\title{
Quantitative Assessment of the Atom Radii of Chemical Elements Based on Spectral Analysis Data
}

\author{
B. L. Aleksandrov \\ Department of Physics \\ Kuban State Agrarian University \\ Krasnodar, Russia \\ alex2e@yandex.ru
}

L. Sh. Makhmudova

Chemical Technology of Oil and Gas Department

M.D. Millionshtchikov Grozny State Oil Technical University

Grozny, Russia

mls66@mail.ru

\begin{abstract}
Atom radii are calculated for chemical elements in different energy states based on photon energy of the emission (absorption) spectrum of atoms.
\end{abstract}

\section{Keywords: atom, radius, emission spectrum, photon}

\section{INTRODUCTION}

The term nanotechnology stands for a set of methods and techniques used to create materials with dimensions in the nanometre scale (at atomic and molecular levels) in order to produce new products with predetermined properties. Other well-known concepts include molecular nanotechnology (MNT) by K. E. Drexler [1-3], submicron nanosystem technology by N. Taniguchi [4], the molecular building block method [5-8] and in-between level nanotechnology. Development of nanotechnology as a scientific theory can be proved only if nanodevices are made available. In case of the molecular building block method, nanodevices are assembled from chemically pre-synthesized molecules (carbon-bearing building blocks that also include nitrogen, boron, etc.) that are capable of forming bonds with each other [5-8]. Such dimensions are typical for the main biological structures: cells, their components (organelles) and molecules, complex inorganic compounds examined in recent scientific publications [9-11]. Taking into account significance and rapid advancement of nanotechnologies, we decided to provide a quantitative assessment of atom radii for different chemical elements in this publication.

A radius of the atom is one of the most important parameters that determines its length and, as a result, the strength of chemical bonds between atoms in molecules, as well as the efficiency of different technological processes. Therefore, quantitative assessment of atom radii for different chemical elements depending on their energy state is significant both from theoretical and practical point of views.

\author{
E. A. Aleksandrova \\ Department of Chemistry \\ Kuban State Agrarian University \\ Krasnodar, Russia \\ alex2e@yandex.ru
}

\author{
Zh. T. Khadisova \\ Chemical Technology of Oil and Gas Department \\ M.D. Millionshtchikov Grozny State Oil Technical \\ University \\ Grozny, Russia \\ Janna h@list.ru
}

\section{DIFFERENT CONCEPTS OF AN ATOMIC RADIUS}

To define a radius of the atom, a number of concepts are applicable depending on the modeling paradigm, classical or quantum mechanical one: atomic, orbital, effective, covalent, or van der Waals radius. In the N. Bohr model of the atom, where electrons travel in defined circular orbits (i.e. energy levels), the radius of the atom is the distance from the center of the nucleus to the furthest electron orbit. An atomic radius is a characteristic of an atom that allows for an approximate estimation of interatomic (inter-nuclear) distances in molecules and crystals $[12,13]$. An orbital radius of an atom is theoretically calculated as a distance from the nucleus to the maximum in the radial probability distribution curve for the electron density in an atom, i.e. it is a characteristic of the free atom sizes [13-15]. For various quantum-chemical calculations, values for orbital radii can differ.

The effective radius of an atom is estimated according to its sphere of action in the compound. It depends on its action manifestation, i.e. it is a characteristic of atoms in molecules bound together in a crystal structure. The radius value depends on the atom environment and interactions, as well as the state of atoms in the crystal structure. It is measured using X-ray data and is based on inter-nuclear distances. Otherwise, electron density distribution maps can be used and the radius is calculated as a distance from the nucleus to the electron density minimum along a bond line.

The concepts of orbital and effective [14, 15] radii are based on solutions to the Schrödinger's equation and assume the wave-particle duality of electrons moving in a hydrogen atom. The radius of the hydrogen atom is determined by atomic orbital types that significantly differ: $s, \mathrm{p}, \mathrm{d}, \mathrm{h}, \mathrm{f}$, etc. Therefore, according to the wave properties of an electron, 
atoms do not have strict boundaries and the concept of the atomic radius implies that $90-98 \%$ of the electron density lies within this sphere. It should be emphasized that the Schrödinger's equation describes the motion of a single electron in a hydrogen atom or a hydrogen-like ion. However, it is currently used for multi-electron atoms as well. For example, B. F. Bratsev [16] considers atom wave function for atom states from helium to argon. To calculate them, he uses the quantum theory of the atom with the Hartree-Fock approximation. During the last decade a number of research papers offered a more critical approach to the Schrödinger's theory [17-19].

Depending on the atom bond type, metal, covalent and van der Waals atomic radii are also distinguished [15]. A metal radius is half the shortest distance between two atoms between atoms in the metal crystal structure. The value depends on the number of neighbors in the structure around the atom in the structure (i.e. coordination number). A covalent radius is half the length of a single chemical bond $\mathrm{X}-\mathrm{X}$, where $\mathrm{X}$ is a nonmetal atom. For halogen, the covalent atomic radius is half the internuclear distance in the $\mathrm{X}_{2}$ molecule.

The size of an atom is determined by its electron shell. However, the shell is not limited to a strict defined surface. The effective radius of the atom is specified by the van der Waals radius. It specifies the minimum possible distancedependent interactions for atoms that belong to different molecules. Thus Van der Waals radius equals half the internuclear distance between the nearest identical atoms that are not connected by chemical bonds, i.e. belong to different molecules. These radii determine the effective size of noble gas atoms [20].

\section{EXISTING Methods For DeterminING AtOM RADII}

In 1911 E. Rutherford experimentally proved the planetary model of the atom, while N. Bohr introduced a theoretical model for it together with a formula for calculating chemical element atom radius [21]:

$$
r_{n}=n^{2} \frac{\hbar^{2} 4 \pi \varepsilon_{0}}{m_{e} Z e^{2}}=0.529 \cdot 10^{-10} \frac{n^{2}}{z},
$$

where $n$ is the main quantum number that can take integer values from 1 to $\infty ; m e_{e}$ is electron mass; $e$ is electron charge; $Z$ is a number of protons in the atom nucleus or the atomic number of a chemical element in the Mendeleev's periodic table; $\varepsilon_{0}$ is an electric constant or a vacuum permittivity constant; $\hbar=\frac{h}{2 \pi} ; h$ is a Planck constant.

As this formula shows, radii of atoms (outermost electron orbits) increase in proportion to the integer squares. For the hydrogen atom $(Z=1)$, the radius of the first electron orbit when $n=1$ is called the Bohr radius and equals $0.529 \cdot 10^{-10} \mathrm{~m}$. This value is considered to be a close approximation of reality. However, there is a drawback to the equation (1): the size of the atom radii of chemical elements increases drastically if $n$ parameter increases. According to it, if $n=\infty$ then $r=\infty$, which makes no physical sense. In addition, in the equation (1) for a valence electron only the net positive charge is considered via $Z$ variable. It does not take into account the distribution of energy as a nucleus interacts with all atom's electrons and electron shielding effect in polyelectronic atoms. Therefore, the equation (1) can actually be used only to calculate the hydrogen atom radius by $n=1 \div 7$.

There is another method for assessing atom radii for chemical elements [12]. It uses a bulk density value for a substance composed of these elements and takes into account the estimated coordination number and crystal lattice structures:

$$
r=\sqrt{\frac{3 \cdot \mu \cdot(1-\mathrm{Kn} a)}{4 \cdot \rho \cdot \mathrm{N} a}}
$$

where $\rho$ is the bulk density of the substance $\left(\frac{\mathrm{kg}}{\mathrm{m}^{3}}\right)$;

$\mu$ is the molar mass $\left(\frac{k g}{m o l e}\right)$;

$K_{n a}$ is interatomic porosity expressed as a decimal fraction;

$\mathrm{Na}$ is the Avogadro's number $\left(\mathrm{Na}=6,022 \cdot 10^{23}\right.$ atoms/mole).

Atom radii for many chemical elements were calculated using the equation (2) by different authors (L. Pauling, N. No. Belov and G. Bokiia and others) and can be found in a book by T. Goronovsky et al. [12] according to their coordination numbers. This method of calculation has its drawback: there is no data on the densities and types of crystal structures, i.e. a reliable estimate for the $K_{n a}$ parameter of substances composed from different chemical elements in different energy states.

In a research [14] it is noted that there exist around 20 systems of values of effective radii for atoms and ions. These systems are based on two established approaches. The first approach assumes that a radius of the oxygen ion is greater than the radius of main cations. It was suggested by A. Lande in the $1920 \mathrm{~s}$ and is based on the additivity principles (i.e. internuclear distance is the sum of the radii of atoms or ions) and densest packing of atoms in crystals (anion spheres touch each other). Anion radius is defined as half the shortest distance between anions. Based on internuclear distances in some selenides and sulfides, radius values were calculated for $\mathrm{S}_{2}-0.183 \mathrm{~nm}, \mathrm{Se}_{2}-0.193 \mathrm{~nm}, \mathrm{Mg}_{2}+(0.076 \mathrm{~nm})$. Later in 1926 V. Goldschmidt created a radius system for ions of all chemical elements. For reference values, he used an anion radius of fluorine $(0,133 \mathrm{~nm})$ and oxygen $(0,132 \mathrm{~nm})$. These values were calculated using a refractometric method by $\mathrm{D}$. Vazasherna in 1923 (based on refraction indices of fluoride in aqueous solutions). Later this system of radii was elaborated by N. Belova, G. Bokiia, L. Pauling, R. Shanon, K. Prewitt and others. In the Pauling system, the $\mathrm{O}_{2}$ radius is $0.140 \mathrm{~nm}$, in the Bokiia-Belov system it is $0.136 \mathrm{~nm}$. 
The second approach was developed by W. L. Bragg in 1920 as he analyzed crystal structure by means of X-ray. As a reference value, he uses the radius of sulfidion $(0.103 \mathrm{~nm})$ that was defined based on internuclear distance $S-S(0.205 \mathrm{~nm})$ in pyrite $\left(\mathrm{FeS}_{2}\right)$ via radiographic measurement techniques. The principles of calculating radii of ions for all other elements stayed the same as in the first approach. However, he assumed the radii of oxygen and sulfur anions to be smaller than the radii of cations, i.e. the densest packing is formed by cations while anions fill in the voids.

Radius systems are constantly refined and revised. In the periodic table of chemical elements, values of the orbital radii for atoms are often specified. They are results of theoretical calculations of electron density distribution in isolated atoms. These values usually provide the foundation to establish patterns of atom radius change between chemical element of different groups and periods in the periodic table. For example, data on the atomic radius values can be found in a reference book Physical quantities [22]. However, researches made by different authors, as well as data from the periodic table, do not cover a lot of chemical elements when it comes to their atom radii (for example, promethium, astatine, elements from americium to einsteinium and mendelevium). Therefore, the quantitative assessment of atom radii for chemical elements based on spectral analysis across the whole energy range of atoms significantly expands the possibilities for researching and solving this issue.

\section{VAlidation Of A New Method Of Quantitative ASSESSMENT OF ATOM RADII FOR CHEMICAL ELEMENTS DEPENDING ON THEIR ENERGY STATE}

A research [23] for the first time offers and validates the use of a mathematical expression for quantitative assessment of atom radii for chemical elements in various energy states based on spectral analysis data. It uses a proton-electronphoton model of the atom structure of the atom that was researched in $[18,19]$. It takes into account that photons present in the atom have a definite emission-absorption spectrum, which is directly related to the problem considered in this paper.

Here, we assume that photons actually exist in the atom structure rotating around external electrons and nucleons. The photon spectrum of a particular atom is determined by the presence of photons forming a photon field around each charged particle of the atom. Each electron of an atom is characterized by a specific photon spectrum.

The atom spectrum is the spectrum of all photons emitted or absorbed by the valence (least bound) electron of a given atom. The atom absorbs the same spectrum as the emitted one. Therefore, spectral analysis can be used to determine the presence of certain elements in a substance. In accordance with this principle, hydrogen has six series in the spectrum due to photon emission-absorption. They represent six photon orbitals around the hydrogen atom electron. The presence of a photon electromagnetic field around all charged particles in an atom and interaction of electrons with an atom nucleus via fields stabilizes of the atom. Otherwise, according to the laws of electrodynamics, electrons moving in a circular orbit with centripetal acceleration would have been continuously losing energy and as a result would have fallen to the nucleus. This view conforms with the Bohr's theory in the part where an electron moves in one circular orbit until it jumps to another. However, Bohr states that "the emission of photons occurs when electrons jump from one energy level to another," i.e. it is the transition of electrons that is initial. We cannot agree with Bohr's viewpoint. Since a photon is initial in the emission process, then it is the photons that are absorbed or emitted by the atom for it to be stable in its environment. Researches [23, 24] show that when photons are emitted and $e^{-}$jumps to an orbit closer to the nucleus, the emitted photons create a torque moment for $e^{-}$to rotate around the atom nucleus. This allowed authors to validate the mathematical expression of the electron rotation radius by emission of a photon of the corresponding frequency and energy.

$$
\begin{aligned}
r_{i}= & \frac{\xi \cdot e^{2}}{8 \pi^{2} \cdot \varepsilon_{0} h \cdot v_{i} \quad}=\frac{\xi \cdot e^{2} \cdot \lambda_{i}}{8 \pi^{2} \cdot \varepsilon_{0} h \cdot c} \quad \text { therefore, } \\
& r_{i}=5,55 \cdot 10^{4} \frac{\xi}{v_{i}}=1,85 \cdot 10^{-4} \cdot \xi \cdot \lambda_{i}(3) \\
& e \text { is electron charge }\left(e=1,6 \cdot 10^{-19} \mathrm{C}\right) ; \\
& \varepsilon_{0} \text { is a vacuum permittivity constant }\left(\varepsilon_{0}=8,85 \cdot 10^{-12} \frac{\mathrm{F}}{\mathrm{m}}\right) ;
\end{aligned}
$$

$h$ is a Planck constant $\left(h=6,626 \cdot 10^{-34} \mathrm{~J} \cdot \mathrm{s}\right)$;

$\lambda_{i}, v_{i}$ is length and frequency of the wave emitted by the atom of the $i$-th photon in the emission spectrum;

$c-$ is the speed of light $\left(c=2,997 \cdot 10^{8} \mathrm{~m} / \mathrm{s}\right)$;

$\xi=\frac{F_{i o n}}{F_{c}}$ is the ratio of atom ionization force $\left(F_{\text {ion }}\right)$ to the coulombic attraction force $\left(F_{\mathrm{c}}\right)$ of the electron and a nucleus assuming that only one proton of the nucleus has influence. The ratio value varies from 0.45 to 1.15 . The most probable value is within the range of $0.75-0.8[23,24]$. Therefore, for most elements when calculating the force of electron-nucleus interaction, one can use the value $\xi=0.75$ or a specific $\xi$ value calculated for the element.

This approach can be used to assess atom radii for chemical elements across the entire energy range, i.e. in a wide range of temperatures. Thus, using the proposed method, we have calculated the atom radii for all chemical elements across their entire energy state range, which distinguishes this work from those of other authors (V. Brattsev [16], A. Radtsig and V. Shustriakov [22], L. Pauling, N. Belov and G. Bokiiu [12] and others).

\section{PRACTICAL IMPlementation Of Quantitative ASSESSMENT OF ATOM RADII FOR CHEMICAL ELEMENTS BASED ON SPECTRAL ANALYSIS DATA}

As examples of assessment of atom radii of chemical elements in different energy states, Table 1 offers results of radius calculations for a mercury atom using formula (3). The 
calculations were made for all wavelengths of the emitted photons according to the tables of spectral lines [25].

$$
\begin{aligned}
& \xi=\frac{F_{\text {ion }}}{F_{c}}=\frac{e \cdot \varphi_{\text {ion }} \cdot 4 \pi \varepsilon_{0} r^{2}}{r \cdot e^{2}}=\frac{\varphi_{\text {ion }} \cdot 4 \pi \varepsilon_{0} r}{e} \\
& \xi=\frac{10.437 \cdot 4 \cdot 3.14 \cdot 8.85 \cdot 10^{-12} \cdot 1.57 \cdot 10^{-10}}{1.6 \cdot 10^{-19}}=1.14
\end{aligned}
$$

\begin{tabular}{|c|c|c|c|c|c|c|c|}
\hline $\begin{array}{c}\text { Emission wavelength } \\
\lambda_{i}, \AA\end{array}$ & $\begin{array}{l}\text { Atom } \\
\text { radius } \\
r . A\end{array}$ & $\begin{array}{c}\text { Emission wavelength } \\
\lambda_{i}, A\end{array}$ & $\begin{array}{l}\text { Atom } \\
\text { radius } \\
r . A\end{array}$ & $\begin{array}{c}\text { Emission wavelength } \\
\lambda_{i}, A\end{array}$ & $\begin{array}{l}\text { Atom } \\
\text { radius } \\
r, \triangle\end{array}$ & $\begin{array}{c}\text { Emission wavelength } \\
\lambda_{i}, A\end{array}$ & $\begin{array}{c}\text { Atom } \\
\text { radius } \\
\text { A A }\end{array}$ \\
\hline 1 & 2 & 1 & 2 & 1 & 2 & 1 & 2 \\
\hline 45122.04 & 9.4969 & 11206.0 & 2.3585 & 4991.5 & 1.0506 & 2803.47 & 0.5900 \\
\hline 39283.61 & 8.2681 & 11176.8 & 2.3524 & 4916.07 & 1.0347 & 2799.76 & 0.5893 \\
\hline 36303.03 & 7.6407 & 10715.5 & 2.2553 & 4890.27 & 1.0292 & 2759.71 & 0.5808 \\
\hline 32148.06 & 6.7663 & 10432.0 & 2.1956 & 4827.1 & 1.0160 & 2752.78 & 0.5794 \\
\hline 23253.07 & 4.8941 & 10423.5 & 2.1938 & 4358.33 & 0.9173 & 2699.51 & 0.5682 \\
\hline 22493.28 & 4.7341 & 10359.5 & 2.1804 & 4347.49 & 0.9150 & 2698.83 & 0.5680 \\
\hline 19700.17 & 4.1463 & 10333.0 & 2.1748 & 4343.63 & 0.9142 & 2655.13 & 0.5588 \\
\hline 18130.38 & 3.8158 & 10298.2 & 2.1675 & 4339.22 & 0.9133 & 2653.68 & 0.5585 \\
\hline 17436.18 & 3.6698 & 10229.6 & 2.1530 & 4108.05 & 0.8646 & 2652.04 & 0.5582 \\
\hline 17329.41 & 3.6473 & 10139.79 & 2.1341 & 4077.83 & 0.8583 & 2625.19 & 0.5525 \\
\hline 17213.20 & 3.6229 & 10129.7 & 2.1320 & 4046.56 & 0.8517 & 2603.15 & 0.5479 \\
\hline 17206.15 & 3.6214 & 10121.2 & 2.1302 & 3906.37 & 0.8222 & 2576.29 & 0.5422 \\
\hline 17198.67 & 3.6198 & 7728.82 & 1.6267 & 3801.66 & 0.8001 & 2536.52 & 0.5339 \\
\hline 17116.75 & 3.6025 & 7091.86 & 1.4926 & 3704.17 & 0.7796 & 2534.76 & 0.5335 \\
\hline 17109.93 & 3.6011 & 7081.9 & 1.4905 & 3701.44 & 0.7790 & 2483.82 & 0.5228 \\
\hline 17072.79 & 3.5933 & 6907.46 & 1.4538 & 3663.28 & 0.7710 & 2482.71 & 0.5225 \\
\hline 16942.00 & 3.5658 & 6716.43 & 1.4136 & 3662.88 & 0.7709 & 2482.00 & 0.5224 \\
\hline 16933.27 & 3.5639 & 6234.4 & 1.3121 & 3654.84 & 0.7692 & 2464.06 & 0.5186 \\
\hline 16920.16 & 3.5611 & 6072.72 & 1.2781 & 3650.15 & 0.7682 & 2399.73 & 0.5051 \\
\hline 16881.48 & 3.5530 & 5871.97 & 1.2359 & 3341.48 & 0.7033 & 2399.35 & 0.5050 \\
\hline 15295.82 & 3.2193 & 5859.25 & 1.2332 & 3144.48 & 0.6618 & 2379.99 & 0.5009 \\
\hline 13950.55 & 2.9361 & 5803.78 & 1.2215 & 3135.76 & 0.6599 & 2378.32 & 0.5006 \\
\hline 13673.51 & 2.8778 & 5790.66 & 1.2188 & 3131.84 & 0.6592 & 2374.02 & 0.4997 \\
\hline 13570.21 & 2.8561 & 5789.66 & 1.2185 & 3131.55 & 0.6591 & 2352.48 & 0.4951 \\
\hline 13505.58 & 2.8425 & 5769.60 & 1.2143 & 3125.67 & 0.6579 & 2345.43 & 0.4936 \\
\hline 13468.38 & 2.8347 & 5675.86 & 1.1946 & 3027.49 & 0.6372 & 2323.20 & 0.4890 \\
\hline 13426.57 & 2.8259 & 5549.63 & 1.1680 & 3025.61 & 0.6368 & 2302.06 & 0.4845 \\
\hline 13209.95 & 2.7803 & 5460.73 & 1.1493 & 3023.48 & 0.6363 & 2002.00 & 0.4214 \\
\hline 12071.7 & 2.5407 & 5384.63 & 1.1333 & 3021.50 & 0.6359 & 1972.94 & 0.4152 \\
\hline 12020.0 & 2.5298 & 5354.05 & 1.1269 & 2967.28 & 0.6245 & 1849.50 & 0.3893 \\
\hline 11977.3 & 2.5209 & 5290.74 & 1.1135 & 2925.41 & 0.6157 & 1832.60 & 0.3857 \\
\hline 11769.1 & 2.4770 & 5218.9 & 1.0984 & 2893.59 & 0.6090 & 1775.68 & 0.3737 \\
\hline 1168.7 & 2.4601 & 5137.94 & 1.0814 & 2856.94 & 0.6013 & 1774.90 & 0.3736 \\
\hline 11491.7 & 2.4186 & 5120.63 & 1.0777 & 2847.83 & 0.5994 & 1250.59 & 0.1847 \\
\hline 11372.5 & 2.3935 & 5102.71 & 1.0740 & 2806.77 & 0.5907 & & \\
\hline 11287.4 & 2.3756 & 5025.64 & 1.0577 & 2804.43 & 0.5902 & & \\
\hline
\end{tabular}

TABLE I. CALCULATION OF MERCURY ATOM RADII ACROSS THE WHOLE RANGE OF ENERGY STATES

To confirm validity of the calculated results of mercury atom radii across a wide range of energy states, they are compared to mercury atoms radii calculated based on density values at three temperature states according to the data provided by P. Babicheva [22]. Density of mercury $\rho$ :

at $t=-39.9^{\circ} \mathrm{C}$ it is equal to $14.193 \cdot 10^{3} \frac{\mathrm{kg}}{\mathrm{m}^{3}}$;

at $t=0^{\circ} \mathrm{C}$ it is equal to $13.59503 \cdot 10^{3} \frac{\mathrm{kg}}{\mathrm{m}^{3}}$, where $\varphi_{\text {ion }}=10.437 \mathrm{eV}$ is ionization potential for the mercury atom; $\mathrm{e}=1.6 \cdot 10^{-19} \mathrm{C} ; \varepsilon_{0}=8.85 \cdot 10^{-12} \frac{\mathrm{F}}{\mathrm{m}} \cdot r=1.57 \cdot 10^{-10}$ $\mathrm{m}$ is the mercury atom radius according to the data by Radtsig and V. Shustriakov [22]. 
result: $r=3.2503 \cdot 10^{-10} m$ at $t=-39.9^{\circ} \mathrm{C}, r=3.321 \cdot 10^{-10} m$ at $t=0^{\circ} \mathrm{C}, r=3.597769 \cdot 10^{-10} \mathrm{~m}$ at $t=800^{\circ} \mathrm{C}$. The mercury atom radii calculated using density values at three temperature states are consistent with the quantitative assessment of the mercury atom radii of the wavelength range $\lambda=(17109.93 \div 15295.82)$ $\AA$, (the spectrum used in calculations is $142 \div 15295.82$ ) $\AA$, (the spectrum used in calculations corresponds to 142 photons, with the range of emitted waves from $\lambda_{\max }=45122.04 \AA$ to $\left.\lambda_{\min }=1250,59 \AA\right)$. The radius of the mercury atom in this wavelength range varies from $r_{\max }=9.4969 \AA$ to $r_{\min }=0.1847$ Á.

TABLE II. CALCULATION OF MERCURY ATOM RADII ACROSS THE WHOLE RANGE OF ENERGY STATES (PART 1)

\begin{tabular}{|c|c|c|c|c|c|c|c|c|c|c|}
\hline \multirow[b]{2}{*}{ № } & \multirow[b]{2}{*}{ Element } & \multicolumn{4}{|c|}{ I Electron } & \multicolumn{5}{|c|}{ Atom radius, $\mathbf{A}$} \\
\hline & & $\lambda_{\text {min }}, \dot{A}$ & $\lambda_{\max }, \dot{A}$ & $\dot{\Gamma}_{\min }, \dot{A}$ & $\dot{\Gamma}_{\max }, \dot{A}$ & $\vec{\Xi} \bar{\Xi}$ & 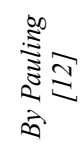 & 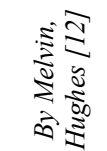 & 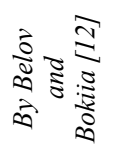 & 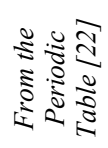 \\
\hline 1 & 2 & 3 & 4 & 5 & 6 & 7 & 8 & 9 & 10 & 11 \\
\hline 1 & Hydrogen & 911.754 & 388592.7 & 0.1683 & 71.734 & 0.79 & 0.31 & 0.3707 & 0.46 & --- \\
\hline 2 & Helium & 320.392 & 21132.04 & 0.0473 & 3.1212 & 0.49 & --- & 0.53 & 1.22 & --- \\
\hline 3 & Lithium & 2333.93 & 68580.17 & 0.3447 & 10.129 & 1.57 & 1.34 & 1.52 & 1.55 & 1.57 \\
\hline 4 & Beryllium & 1426.11 & 31778.70 & 0.2106 & 4.6937 & 1.13 & 1.07 & 1.113 & 1.13 & 1.13 \\
\hline 5 & Bohr & 993.38 & 36010.65 & 0.1467 & 5.3187 & 0.97 & 0.89 & 0.795 & 0.91 & 0.97 \\
\hline 6 & Carbon & 945.191 & 25842.20 & 0.1396 & 3.8168 & 0.77 & 0.77 & 0.771 & 0.77 & 0.77 \\
\hline 7 & Nitrogen & 612.883 & 18751.01 & 0.0905 & 2.7695 & 0.71 & 0.70 & 0.547 & 0.71 & 0.71 \\
\hline 8 & Oxygen & 679.202 & 26173.56 & 0.1003 & 3.8658 & 0.74 & 0.66 & 0.6037 & --- & 0.74 \\
\hline 9 & Fluorine & 680.698 & 11557.17 & 0.1005 & 1.7069 & 0.64 & 0.64 & 0.709 & --- & --- \\
\hline 10 & Neon & 576.00 & 40575.72 & 0.0850 & 5.9930 & 1.6 & --- & 1.60 & 1.60 & 1.60 \\
\hline 11 & Sodium & 2464.77 & 40431.88 & 0.3640 & 5.9717 & 1.86 & 1.54 & 1.858 & 1.89 & 1.86 \\
\hline 12 & Magnesium & 1625.22 & 26392.90 & 0.2400 & 3.8982 & 1.6 & 1.40 & 1.599 & 1.60 & 1.60 \\
\hline 13 & Aluminum & 1181.807 & 21163.75 & 0.1745 & 3.1258 & 1.43 & 1.26 & 1.432 & 1.43 & 1.43 \\
\hline 14 & Silicon & 1255.276 & 26644.44 & 0.1854 & 3.9353 & 1.18 & 1.17 & 1.176 & 1.34 & 1.18 \\
\hline 15 & Phosphorus & 1241.49 & 20590.73 & 0.1833 & 3.0412 & 1.3 & 1.10 & 0.947 & 1.30 & 1.30 \\
\hline 16 & Sulfur & 1049.82 & 34270.76 & 0.1550 & 5.0617 & 1.04 & 1.04 & 1.02 & --- & 1.04 \\
\hline 17 & Chlorine & 969.917 & 40532.16 & 0.1432 & 5.9866 & --- & 0.99 & 0.994 & --- & --- \\
\hline 18 & Argon & 797.744 & 40880.07 & 0.1178 & 6.0379 & 1.92 & --- & 1.92 & 1.92 & 1.92 \\
\hline 19 & Potassium & 653.31 & 85100.0 & 0.0964 & 12.569 & 2.36 & --- & 2.272 & 2.36 & 2.36 \\
\hline 20 & Calcium & 2150.78 & 19932.94 & 0.3176 & 2.9440 & 1.97 & --- & 1.974 & 1.97 & 1.97 \\
\hline 21 & Scandium & 2706.78 & 7800.44 & 0.3997 & 1.1521 & 1.62 & --- & --- & 1.64 & 1.62 \\
\hline 22 & Titanium & 2273.28 & 11403.89 & 0.3357 & 1.6843 & 1.46 & --- & 1.44 & 1.46 & 1.46 \\
\hline 23 & Vanadium & 1957.90 & 11107.7 & 0.2891 & 1.6406 & 1.31 & --- & 1.32 & 1.34 & 1.31 \\
\hline 24 & Chrome & 1999.95 & 11610.48 & 0.2953 & 1.7148 & 1.27 & 1.25 & 1.249 & 1.27 & 1.27 \\
\hline 25 & Manganese & 1996.06 & 17607.50 & 0.2948 & 2.6006 & 1.30 & --- & 1.366 & 1.30 & 1.30 \\
\hline 26 & Iron & 1855.58 & 11973.07 & 0.2740 & 1.7684 & 1.26 & --- & 1.241 & 1.26 & 1.26 \\
\hline 27 & Cobalt & 1847.89 & 11630.93 & 0.2729 & 1.7178 & 1.25 & 1.24 & 1.246 & 1.24 & 1.25 \\
\hline 28 & Nickel & 2014.25 & 18040.6 & 0.2975 & 2.6646 & 1.24 & 1.24 & 1.246 & 1.24 & 1.24 \\
\hline 29 & Copper & 1713.36 & 18229.0 & 0.2530 & 2.6924 & 1.28 & 1.35 & 1.278 & 1.28 & 1.28 \\
\hline 30 & Zinc & 809.92 & 7799.37 & 0.1196 & 1.1519 & 1.37 & 1.31 & 1.333 & 1.39 & 1.37 \\
\hline 31 & Gallium & 1505.6 & 12109.93 & 0.2223 & 1.7886 & 1.39 & --- & 1.221 & 1.39 & 1.39 \\
\hline 32 & Germanium & 1143.25 & 20673.64 & 0.1688 & 3.0534 & 1.39 & --- & 1.149 & 1.39 & 1.39 \\
\hline 33 & Arsenic & 1515.48 & 10888.82 & 0.2238 & 1.6082 & 1.48 & 1.21 & 1.248 & 1.48 & 1.48 \\
\hline 34 & Selenium & 1377.98 & 10386.28 & 0.2035 & 1.5340 & 1.6 & & 1.16 & 1.60 & 1.60 \\
\hline 35 & Bromine & 1101.5 & 22865.65 & 0.1629 & 3.3772 & 1.19 & 1.14 & 1.141 & --- & 1.19 \\
\hline 36 & Krypton & 862.36 & 30979.22 & 0.1273 & 4.5756 & 1.98 & --- & 1.97 & 1.98 & 1.98 \\
\hline 37 & Rubidium & 3023.66 & 27905.37 & 0.4465 & 4.1216 & 2.48 & --- & 2.475 & 2.48 & 2.48 \\
\hline 38 & Strontium & 2428.1 & 30110.0 & 0.3586 & 4.4472 & 2.15 & --- & 2.151 & 2.15 & 2.15 \\
\hline 39 & Yttrium & 2813.64 & 10683.4 & 0.4155 & 1.5779 & 1.81 & --- & --- & 1.81 & 1.81 \\
\hline 40 & Zirconium & 2285.25 & 10210.44 & 0.3375 & 1.5080 & 1.6 & --- & 1.59 & 1.60 & 1.60 \\
\hline 41 & Niobium & 2211.46 & 10563.7 & 0.3266 & 1.5602 & 1.45 & --- & ---- & 1.45 & 1.45 \\
\hline 42 & Molybdenum & 2471.97 & 8389.32 & 0.3651 & 1.2391 & 1.40 & 1.40 & 1.363 & 1.39 & 1.40 \\
\hline
\end{tabular}


TABLE III. CALCULATION OF MERCURY ATOM RADII ACROSS THE WHOLE RANGE OF ENERGY STATES (PART 2)

\begin{tabular}{|c|c|c|c|c|c|c|c|c|c|c|}
\hline \multirow[b]{2}{*}{ № } & \multirow[b]{2}{*}{ Element } & \multicolumn{4}{|c|}{ I Electron } & \multicolumn{5}{|c|}{ Atom radius, Á } \\
\hline & & $\lambda_{\text {min }}, \dot{A}$ & $\lambda_{\max }, \dot{A}$ & $\dot{\Gamma}_{\min }, \dot{A}$ & $\dot{\Gamma}_{\max }, \dot{A}$ & 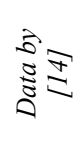 & 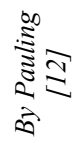 & 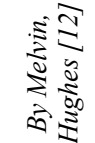 & 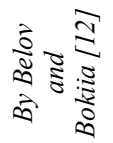 & 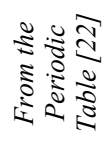 \\
\hline 43 & Technetium & 2376.05 & 8829.8 & 0.3509 & 1.3041 & 1.36 & --- & --- & 1.39 & 1.36 \\
\hline 44 & Ruthenium & 2076.43 & 8264.95 & 0.3066 & 1.2207 & 1.34 & --- & 1.34 & 1.34 & 1.34 \\
\hline 45 & Rhodium & 2264.14 & 9757.11 & 0.3344 & 1.4411 & 1.37 & --- & 1.35 & 1.34 & 1.37 \\
\hline 46 & Palladium & 1890.41 & 10890.26 & 0.2792 & 1.6084 & 1.37 & 1.37 & 1.376 & 1.37 & 1.37 \\
\hline 47 & Silver & 1507.37 & 39951.0 & 0.2226 & 5.9007 & 1.44 & 1.53 & 1.445 & 1.44 & 1.44 \\
\hline 48 & Cadmium & 701.20 & 39087.0 & 0.1035 & 5.7731 & 1.56 & 1.48 & 1.49 & 1.56 & 1.56 \\
\hline 49 & Indium & 1648.0 & 16748.0 & 0.2434 & 2.4736 & 1.66 & --- & 1.626 & 1.66 & 1.66 \\
\hline 50 & Tin & 1804.6 & 13608.0 & 0.2665 & 2.0099 & 1.58 & 1.40 & 1.40 & 1.58 & 1.58 \\
\hline 51 & Antimony & 1456.62 & 12116.06 & 0.2151 & 1.7895 & 1.61 & 1.41 & 1.45 & 1.61 & 1.61 \\
\hline 52 & Tellurium & 1700.0 & 10149.2 & 0.2510 & 1.4990 & 1.7 & $\begin{array}{ll}-- \\
\end{array}$ & 1.35 & 1.70 & 1.70 \\
\hline 53 & Iodine & 1259.15 & 16038.15 & 0.1859 & 2.3688 & 1.36 & 1.33 & 1.3313 & --- & 1.36 \\
\hline 54 & Xenon & 952.1 & 26511.0 & 0.1406 & 3.9156 & 2.18 & --- & 2.18 & --- & 2.18 \\
\hline 55 & Cesium & 3288.61 & 68070.0 & 0.4857 & 10.054 & 2.62 & --- & 2.655 & 2.68 & 2.62 \\
\hline 56 & Barium & 2133.58 & 30933.8 & 0.3151 & 4.5689 & 2.24 & --- & 2.174 & 2.21 & 2.24 \\
\hline 57 & Lanthanum & 3215.81 & 10612.56 & 0.4749 & 1.5674 & 1.87 & --- & 1.87 & 1.87 & 1.87 \\
\hline 58 & Cerium & 3437.81 & 8891.2 & 0.5077 & 1.3132 & 1.82 & --- & 1.825 & 1.83 & 1.82 \\
\hline 59 & Praseodymium & 2488.75 & 8854.05 & 0.3675 & 1.3077 & 1.82 & --- & $\begin{array}{l}-- \\
-\end{array}$ & 1.82 & 1.82 \\
\hline 60 & Neodymium & 4337.22 & 6846.72 & 0.6406 & 1.0112 & 1.82 & --- & --- & 1.82 & 1.82 \\
\hline 61 & Promethium & 2502.12 & 8335.63 & 0.3695 & 1.2311 & --- & --- & --- & --- & --- \\
\hline 62 & Samarium & 3687.88 & 8913.66 & 0.5446 & 1.3165 & 1.8 & --- & --- & 1.81 & 1.80 \\
\hline 63 & Europium & 2709.99 & 7887.98 & 0.4002 & 1.1650 & 2.04 & --- & --- & 2.02 & 2.04 \\
\hline 64 & Gadolinium & 2892.08 & 8668.63 & 0.4271 & 1.2803 & 1.79 & --- & --- & 1.79 & 1.79 \\
\hline 65 & Terbium & 3558.77 & 6896.37 & 0.5256 & 1.0185 & 1.77 & --- & ---- & 1.77 & 1.77 \\
\hline 66 & Dysprosium & 2585.3 & 10523.39 & 0.3818 & 1.5543 & 1.77 & --- & --- & 1.77 & 1.77 \\
\hline 67 & Holmium & 3424.11 & 8915.98 & 0.5057 & 1.3168 & 1.76 & --- & --- & 1.76 & 1.76 \\
\hline 68 & Erbium & 3368.18 & 9927.1 & 0.4974 & 1.4662 & 1.76 & --- & 1.86 & 1.75 & 1.76 \\
\hline 69 & Thulium & 2596.49 & 8017.9 & 0.3835 & 1.1842 & 1.75 & --- & ---- & 1.74 & 1.75 \\
\hline 70 & Ytterbium & 2464.5 & 10770.1 & 0.3640 & 1.5907 & 1.93 & --- & --- & 1.93 & 1.93 \\
\hline 71 & Lutetium & 2685.08 & 24170.0 & 0.3965 & 3.5699 & ---- & --- & --- & 1.74 & --- \\
\hline 72 & Hafnium & 2845.83 & 10637.93 & 0.4203 & 1.5712 & 1.59 & --- & 1.61 & 1.59 & 1.59 \\
\hline 73 & Tantalum & 2396.3 & 10099.41 & 0.3539 & 1.4916 & 1.46 & --- & --- & 1.46 & 1.46 \\
\hline 74 & Tungsten & 2098.25 & 8613.26 & 0.3099 & 1.2721 & 1.41 & --- & 1.371 & 1.40 & 1.41 \\
\hline 75 & Rhenium & 1716.45 & 10639.45 & 0.2535 & 1.5714 & 1.37 & ---- & (--- & 1.37 & 1.37 \\
\hline 76 & Osmium & 2001.45 & 7602.95 & 0.2956 & 1.1229 & 1.36 & --- & 1.338 & 1.35 & 1.36 \\
\hline 77 & Iridium & 2010.63 & 7183.71 & 0.2969 & 1.0610 & 1.36 & --- & ---- & 1.35 & 1.36 \\
\hline 78 & Platinum & 1969.69 & 8762.47 & 0.2909 & 1.2942 & 1.39 & 1.38 & 1.388 & 1.38 & 1.39 \\
\hline 79 & Gold & 1578.24 & 9391.7 & 0.2344 & 1.3871 & 1.44 & 1.50 & 1.442 & 1.44 & 1.44 \\
\hline 80 & Mercury & 1250.59 & 45122.04 & 0.1847 & 6.6645 & 1.57 & 1.48 & 1.503 & 1.60 & 1.57 \\
\hline 81 & Thallium & 1489.65 & 21802.17 & 0.2200 & 3.2201 & 1.71 & --- & 1.704 & 1.71 & 1.71 \\
\hline 82 & Lead & 1722.66 & 10969.53 & 0.2544 & 1.6201 & 1.75 & 1.46 & 1.75 & 1.75 & 1.75 \\
\hline 83 & Bismuth & 1489.94 & 22554.2 & 0.2200 & 3.3312 & 1.82 & 1.51 & 1.548 & 1.82 & 1.82 \\
\hline 84 & Polonium & 1919.4 & 9374.8 & 0.2834 & 1.3846 & 1.19 & --- & --- & --- & 1.19 \\
\hline 85 & Astatin & 2162.25 & 2244.01 & 0.3193 & 0.3314 & --- & --- & --- & --- & --- \\
\hline 86 & Radon & 1451.56 & 9327.02 & 0.2143 & 1.3776 & 1.34 & --- & --- & --- & 1.34 \\
\hline 87 & Francium & & & & & --- & --- & --- & 2.80 & --- \\
\hline 88 & Radium & 3101.8 & 9932.21 & 0.4581 & 1.4669 & --- & --- & --- & 2.35 & --- \\
\hline 89 & Actinium & 2968.82 & 7866.10 & 0.4384 & 1.1618 & --- & --- & --- & 2.03 & --- \\
\hline 90 & Thorium & 2891.25 & 10875.05 & 0.4270 & 1.6062 & 1.80 & --- & --- & 1.80 & 1.80 \\
\hline 91 & Protactinium & 2522.61 & 4433.44 & 0.3725 & 0.6548 & --- & --- & --- & 1.62 & --- \\
\hline 92 & Uranium & 3027.66 & 21911.0 & 0.4471 & 3.2362 & 1.20 & --- & 1.49 & 1.53 & 1.20 \\
\hline 93 & Neptunium & 2655.0 & 4363.8 & 0.3921 & 0.6445 & --- & --- & --- & 1.50 & --- \\
\hline 94 & Plutonium & 2477.0 & 21126.0 & 0.3953 & 3.1203 & --- & --- & --- & 1.62 & --- \\
\hline 95 & Americium & 2953.71 & 8497.82 & 0.4362 & 1.2551 & ---- & ---- & --- & --- & ---- \\
\hline 96 & Curium & 2516.0 & 4989.9 & 0.3716 & 0.7370 & --- & --- & --- & --- & --- \\
\hline 97 & Berkelium & 3412.01 & 3916.24 & 0.5039 & 0.5784 & --- & --- & --- & --- & --- \\
\hline 98 & Californium & 3706.4 & 4335.2 & 0.5474 & 0.6403 & --- & --- & --- & --- & --- \\
\hline 99 & Einsteinium & 3065.4 & 6539.71 & 0.4527 & 0.9659 & ---- & ---- & --- & --- & ---- \\
\hline 100 & Fermium & --- & --- & --- & --- & --- & --- & --- & --- & --- \\
\hline 101 & Mendelevium & --- & --- & ---- & --- & --- & --- & --- & --- & --- \\
\hline
\end{tabular}


In the same way it is possible to calculate atom radii for different elements given that their density and a crystal lattice type, which determines the interatomic porosity, are known. A similar method for calculating the radii of atoms was used by Pauling, Melvin-Hughes, Belov and Bokia [12], whose data for many elements slightly differ, probably since they used different values of the coordination number. However, it is difficult to calculate atom radii for different elements using values of their bulk density in a wide range of temperatures (energy states) since there are no data on their density and crystal lattice types under these conditions.

The formula (3) was used to assess the minimum and the maximum atom radii for all chemical elements based on threshold values of wavelengths of photon emission (absorption) and spectrum data for these chemical elements $[25,26]$. The calculated results of threshold values of the atom radii are shown in Table 2.

If analyzed, this table shows that for $75 \%$ of elements data on atom radii provided by different authors fit into the range $\left(r_{\min }-r_{\max }\right)$, which is calculated using using threshold values of wavelengths of photon emission-absorption. In comparison to others, for the first 18 elements more recent information on the atom spectrum was used. It allowed for more objective data on their atom radius threshold values.

The greatest number of discrepancies between values calculated based on spectral analysis data $\left(r_{\min }-r_{\max }\right)$ and data on the atom radii of elements provided by other authors (Table 2 ) is found for lanthanoid elements. It can be explained by the fact that the spectral analysis data on these and a number of other elements are not complete enough. Such conclusion is confirmed by comparing threshold values of wavelengths for emission-absorption from earlier [25] and later [26] publications for the first 18 elements.

This approach allows for objective assessment of atom radii of chemical elements across the entire range of their energy state, i.e. a wide range of temperatures. Thus, using the proposed method, we have calculated the atom radii for all chemical elements across their entire energy state range, which distinguishes this work from those of other authors (V. Brattsev [16], A. Radtsig and V. Shustriakov [22], L. Pauling [12], N. V. Belov and G. [12], etc.), the data of which correspond to a certain narrow range of temperature corresponding to normal conditions.

\section{CONCLUSION}

1. In this research we provided assessment of the threshold values range ( $\mathrm{rmin}$ - rmax) of atom radii for all chemical elements of the periodic table. It is shown that data on atom radii calculated using threshold values of wavelengths of photon emission-absorption in most cases coincide with the experimental (reference) provided by different authors. For a number of chemical elements, especially heavy ones, data on the change ranges of the atom radii is published for the first time.

2. There is a satisfactory correspondence between values of atom radii calculated based on emitted photon energy and ones calculated using a traditional approach, i.e. via the substance density. It validates the proposed methodological approach to the assessment of atom radii across their entire energy range.

3. The expected decrease of atom radii by photon emission confirms the existence of a photon electromagnetic field around atom electrons and proton-electron-photon model of the atom.

\section{References}

[1] K. Eric Drexler, "Molecular Engineering: An Approach to the Development of General Capabilities for Molecular Manipulation", Proc. Natl. Acad. Soc., USA, 1981, pp. 5275-5278.

[2] K. Eric Drexler, Engines of Creation: The Coming Era of Nanotechnology. New York, 1986, Ancor Press/Doubleday

[3] K. Eric Drexler, Nanosystems: Molecular Machinery, Manufacturing and Computation, New York: John Wiley and Sons, 1992.

[4] N. Taniguchi, "On the Basic Concept of 'Nano-Technology", Proc. Intl. Conf. Prod. Eng. Tokyo, Part II, 1974.

[5] R. A. Freitas, Nanomedicine, vol. I: Basic Capabilities. Landes Bioscience, 1999.

[6] Ralph C Merkle, Molecular building blocks and development strategies for molecular nanotechnology, Nanotechnology, vol. 11, 2000, pp. 8999.

[7] Chris Phoenix, "Design of a Primitive Nanofactory", Journal of Evolution and Technology, vol. 13-October 2003.

[8] S. I. Sadovnikov, "Nano-structured silver sulfide: synthesis of different forms and application". Advances in chemistry, vol. 87(4), pp.303-327, 2018 .

[9] V. N. Postnov, "From carbon nanostructures to high performance sorbents for chromatographic separation and concentration", Advanced Chemistry, vol. 85(2), pp. 115-130, 2016.

[10] M. Yu. Tsvetkov, V. I. Yusupov, P. S. Timashev, K. M. Golant, N. V. Minaev, V. N. Bagratashvili, "Improving the efficiency of laser liquid etching of optically transparent materials due to laser-induced formation of carbon and silver nanoparticles", Russian nanotechnology, vol.12, no.1-2, pp.69 -71, 2017.

[11] M. E. Burin, A. P. Pushkarev, G. K. Fukin, R. V. Rumyantsev, A. N. Konev, M. N. Bochkarev, "Preparation of EuS, EuSe nanoparticles by thermal decomposition of dithio- and diselenophosphinate complexes of europium", Russian nanotechnology, vol.12, no.1-2, pp.53 -59, 2017.

[12] I. T. Goronovsky, Yu. P. Nazarenko, E. F. Nekryach, Chemistry reference book. Fifth edition. 1987, p.830.

[13] Chemical encyclopedia, vol. 4, 1995, p. 813.

[14] A. T. Serkov, A. A. Serkov, Line spectra emission and orbital radii of a hydrogen atom. Scientific engineering center Uglechimvolokn.

[15] Physical encyclopaedic dictionary, 1984, p. 944.

[16] V. F. Bratsev, Tables of atomic wave functions, 1966, p.192.

[17] A. A. Potapov. Renaissance of the classical atom, 2011, p.440.

[18] B. L. Alexandrov, M. B. Rodchenko, A. B. Alexandrov, The role of photons for physical and chemical phenomena, 2002, p. 543.

[19] B. L. Alexandrov , M. B. Rodchenko, E. A. Alexandrova, The photon theory of chemical bonds and atomic structure, Abstracts Plenary reports, Achievements and prospects of chemical science., vol. 1, p. 5657, September 2007 [XVIII Mendeleev Congress on General and applied chemistry, p.4.94, 2007].

[20] A. I. Volkov, I. M. Zharskoi, Large chemical reference book, 2005, p. 608.

[21] T. I. Trofimova, the Course of physics, 2000, p.542.

[22] S. Grigorieva, E. Z. Meilikhova, Physical quantities. Handbook, 1991, p.1232.

[23] B. L. Alexandrov, M. B. Rodchenko, A Way of measuring of atom radii of chemical elements in different energy states. Russian Federation 
patent №2273058. Registered in The state register of inventions of the Russian Federation on March 27, 2006 with priority of August 30, 2004.

[24] B. L. Aleksandrov B. L., Rodchenko M. B., Calculation of atom radii in different energy states, Abstracts. Plenary report. Fundamental problems of chemical science, vol. 1, p. 360-361,September 2011 [XIX Mendeleev Congress on General and Applied Chemistry, p. 464, 2011].
[25] Tables of spectral lines. Fourth edition, 1977, p. 4.800

[26] A. R. Striganov, G. A. Odintsova, Tables of spectral lines of atoms and ions, 1982, p. 312 